(C) 2016 IEEE. Personal use of this material is permitted. Permission from IEEE must be obtained for all other uses, in any current or future media, including reprinting/republishing this material for advertising or promotional purposes, creating new collective works, for resale or redistribution to servers or lists, or reuse of any copyrighted component of this work in other works. 


\title{
Non-Contact Real-Time Estimation of Intrapulmonary Pressure and Tidal Volume for Chronic Heart Failure Patients
}

\author{
Vinh Phuc Tran, Student Member, IEEE, and Adel Ali Al-Jumaily, Senior Member, IEEE
}

\begin{abstract}
Long-term continuous patient monitoring is required in many health systems for monitoring and analytical diagnosing purposes. It has been recognized that these types of monitoring systems have shortcomings related to patient comfort and/or functionality. Non-contact monitoring systems have been developed to address some of these shortcomings. One of such systems is non-contact physiological vital signs assessments for Chronic Heart Failure (CHF) patients. This paper presents a novel pulmonary ventilation model that defines the relationship between the intrapulmonary pressure and the chest displacement. A novel intrapulmonary pressure and tidal volume estimation algorithm is also proposed. A database consisting of twenty CHF patients with New York Heart Association (NYHA) Heart Failure Classification Class II \& III; whose underwent full Polysomnography (PSG) analysis for diagnosis of sleep apnea, disordered sleep, or both, was selected for the verification of the proposed model and algorithm. The proposed algorithm analyzes the non-contact sensor data and estimate the patient's intrapulmonary pressure and tidal volume. The output of the algorithm is compared with the gold-standard PSG recordings. Across all twenty CHF patients' recordings with mean recorded sleep duration of 7.76 hours, the tidal volume estimation median accuracy achieved $\mathbf{8 3 . 1 3 \%}$ with a median error of $\mathbf{5 7 . 3 2}$ milliliters. A potential application would be non-contact continuous monitoring of intrapulmonary pressure and tidal volume during sleep in the home.
\end{abstract}

\section{INTRODUCTION}

Obstructive sleep apnea (OSA) is a common and potentially lethal sleep disorder affecting at least $4 \%$ of adult males and $2 \%$ of adult females world-wide [1]. Statistics published in 2013 reported that the prevalence of OSA had increased to $10-17 \%$ for males and $3-9 \%$ for females in the United States of America [2]. OSA is the cessation of airflow due to the collapse of the upper airway during sleep [3] and can occur at any age, from infancy to old age. Evidences has indicated that OSA is associated with Chronic Heart Failure (CHF) [4] with an increased prevalence of 2.38 times, independent of other known risk factors [5]. These findings highlighted the needs for long-term continuous sleep monitoring for both OSA and CHF patients.

Doppler radar for physiological vital signs monitoring has been known since 1970's and enormous literatures have been published regarding non-contact assessments of respiratory

*Research supported by ResMed Ltd.

Vinh Phuc Tran is with Healthcare Informatics, ResMed Ltd., and Centre for Health Technologies, Faculty of Engineering \& IT, University of Technology, Sydney (Vinh.P.Tran-1@student.uts.edu.au).

Associate Professor Dr. Adel Ali Al-Jumaily is with Centre for Health Technologies, Faculty of Engineering \& IT, University of Technology, Sydney (Adel.Al-Jumaily@uts.edu.au). and heart rates. However, publications regarding correlations between tidal volume and chest displacement [6, 7] are currently limited. It is also important to indicate that the relationship between tidal volume and chest displacement has not yet been defined in quantitative mathematical equations.

This paper presents a novel pulmonary ventilation model that defines the relationship between the intrapulmonary pressure and the chest displacement. A novel intrapulmonary pressure and tidal volume estimation algorithm is also proposed. This paper is structured as follows: section II describes the non-contact sensor that tracks a person's movement while sleeping using a radiofrequency motion sensor. Based on these signals, the intrapulmonary pressure and tidal volume can be estimated using the proposed model and algorithm as explained in section III and IV. While section $\mathrm{V}$ reports the output of the algorithm as compared with the gold-standard Polysomnography (PSG) recordings from a set of twenty CHF patients. Finally, section VI concludes the work presented in this paper.

\section{Biomotion SENSOR \& PATIENTS DAtABASE}

The SleepMinder ${ }^{\mathrm{TM}}(\mathrm{SM})$ sensor is a ResMed patented non-contact sensor for convenient measurement of sleep and breathing in the home. SM is a dual pulse-Doppler system designed to transmit two short pulses of radio frequency energy at $5.8 \mathrm{GHz}$ and capable of measuring movements at a distance between 0.5-3.0 meters. SM also employs quadrature detection techniques, which leads to two movement signals, called I and Q channels. The I and Q channels are internally filtered by active analog filters at 1.6 $\mathrm{Hz}$ and sampled at $64 \mathrm{~Hz}$. The $64 \mathrm{~Hz}$ samples are then averaged over 4 samples, producing two $16 \mathrm{~Hz}$ channels and saved to the SM memory card in a proprietary binary format.

A database consisting of twenty CHF patients with New York Heart Association (NYHA) Heart Failure Classification Class II \& III was selected for the verification of the proposed model and algorithm. The patients groups are of 2 females and 18 males, who were sequentially admitted in the Royal Brompton Centre for Sleep, London, United Kingdom, for the diagnosis of sleep apnea, disordered sleep, or both. The patients' mean age was 69.05 years, with mean body weight of $82.69 \mathrm{~kg}$, mean body height of $1.72 \mathrm{~m}$, mean BMI of 28.01, and mean recorded sleep duration of 7.76 hours. The consented patients underwent full Polysomnography (PSG) analysis with manually scored by sleep experts. The SM sensor was installed in the sleep laboratory and its biomotion signals were recorded simultaneously with the PSG signals. The SM sensor was positioned facing the patient in line with chest, at a distance of 0.5 meter, and an elevation of 0.5 meter from the edge of the bed. 


\section{PUlmonary Ventilation MOdel}

The process of gas exchange in human body consists of three basic steps: pulmonary ventilation, external respiration, and internal respiration. This paper focuses only on pulmonary ventilation to derive a novel mathematical model that defines the relationship between the intrapulmonary pressure and the chest displacement. The outcome of the derived relationship is the estimation of the intrapulmonary pressure and tidal volume based on the chest displacement. The model takes an engineering approach with the utilization of electronic elements to describe the time-varying differential relationships. The lungs are modeled as a container of certain volume that can hold a certain amount of pressurized gas, which can be described using capacitor " $\mathrm{C}$ ". The resistance and elasticity in the respiratory system are described using resistor " $R$ " and inductor " $L$ ". The model is presented in the Laplace's transform s-domain; this is for mathematical convenience in analyzing the time-varying differential parameters. The novel pulmonary ventilation model is presented below in "Fig. 1":

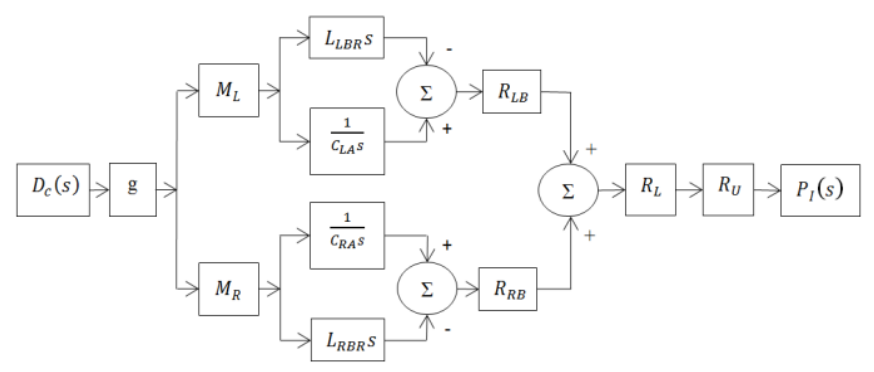

Figure 1. Pulmonary Ventilation Model

The descriptions of the used symbols are as follows:

- $\mathrm{D}_{\mathrm{c}}(\mathrm{s})$ - chest displacement in meter.

- $\mathrm{g}$-standard gravitational constant, i.e., $9.80665 \mathrm{~ms}^{-2}$.

- $\mathrm{M}_{\mathrm{L}} \& \mathrm{M}_{\mathrm{R}}-$ left and right mass portion of the total subject's body mass in kilograms residing at the chest area.

- $\mathrm{C}_{\mathrm{LA}} \& \mathrm{C}_{\mathrm{RA}}$ - left and right lungs volume that can hold a certain amount of pressurized gas.

- $\quad \mathrm{L}_{\mathrm{LBR}} \& \mathrm{~L}_{\mathrm{RBR}}$ - left and right bronchiole and terminal bronchiole elasticity, which cause changes in resistance due to changes in gas pressure.

- $\mathrm{R}_{\mathrm{LB}} \& \mathrm{R}_{\mathrm{RB}}$ - left and right secondary and tertiary bronchus resistance.

- $\mathrm{R}_{\mathrm{L}}$ - larynx and trachea resistance.

- $\mathrm{R}_{\mathrm{U}}$ - upper respiratory system airway resistance, including the nose, pharynx \& associated structures.

- $\quad \mathrm{P}_{\mathrm{I}}(\mathrm{s})$ - intrapulmonary pressure.

From "Fig. 1", the transfer function of the pulmonary ventilation model can be expressed as:

$\frac{P_{I}(s)}{D_{C}(s)}=g R_{L} R_{U}\left(M_{L} R_{L B}\left(\frac{1-L_{L B R} C_{L A} s^{2}}{C_{L A} S}\right)+M_{R} R_{R B}\left(\frac{1-L_{R B R} C_{R A} s^{2}}{C_{R A} s}\right)\right)$
The following assumptions are applied to the pulmonary ventilation model as shown in "Fig. 1":

- The left lung volume that can hold a certain amount of pressurized gas is approximately equal to the right lung volume, denoted by " $\mathrm{C}_{\mathrm{A}}$ ".

- $\quad$ The left bronchiole and terminal bronchiole elasticity are approximately equal to the right bronchiole and terminal bronchiole elasticity, denoted by " $\mathrm{L}_{\mathrm{BR}}$ ".

- The left secondary and tertiary bronchus resistance is approximately equal to the right secondary and tertiary bronchus resistance, denoted by " $\mathrm{R}_{\mathrm{B}}$ ".

Applying the above assumptions simplifies (1) to:

$$
\frac{P_{I}(s)}{D_{C}(s)}=-K\left(\frac{s^{2}-f_{R R}}{s}\right) \quad \text { where } \begin{gathered}
K=g\left(M_{L}+M_{R}\right) L_{B R} R_{B} R_{L} R_{U} \\
f_{R R}=1 / L_{B R} C_{A}
\end{gathered}
$$

The negative sign on the coefficient " $\mathrm{K}$ " in (2) indicates that when the lung volume increases due to the contraction of the diaphragm, which results in the increase of the chest displacement, the intrapulmonary pressure decreases for inspiration to occur, and vice versa for expiration. The bronchiole and terminal bronchiole elasticity " $\mathrm{L}_{\mathrm{BR}}$ " in (2) can be neglected for constant " $\mathrm{K}$ ". This is because the changes in " $\mathrm{L}_{\mathrm{BR}}$ " will impact the intrapulmonary pressure, which in turn reflected through the reduction of the chest displacement magnitude " $\mathrm{D}_{\mathrm{c}}(\mathrm{s})$ ". The secondary and tertiary bronchus resistance " $R_{B}$ ", larynx and trachea resistance " $R_{L}$ ", upper respiratory system airway resistance, including the nose, pharynx and associated structures " $R_{U}$ " in (2), can also be neglected for constant " $K$ ". The changes in these resistances also reflected through the reduction of the chest displacement magnitude " $\mathrm{D}_{\mathrm{c}}(\mathrm{s})$ ". The product of coefficients " $\mathrm{L}_{\mathrm{BR}}$ " and " $\mathrm{C}_{\mathrm{A}}$ " in (2) can be seen as a time constant $(\tau)$, which is the period of the pressure accumulation in the alveoli. The inverse of $(\tau)$, i.e. " $f_{R R}$ ", is, in fact, the frequency of breathing in Hertz. Therefore, " $f_{R R}$ " is approximately equal to the respiratory rate (breaths per minute) divided by 60 seconds.

$f_{R R}=$ Respiratory Rate $/ 60$

The " $\mathrm{s}$ " " term in (2) indicates a second derivative, which in this case is the chest acceleration due to respiration efforts. The chest acceleration multiplied by a constant " $\mathrm{K}$ " indicates both Force (F) and Work (W) are acted on the chest area. According to physics, $\mathrm{W}=\mathrm{mgh}(\mathrm{Nm})$, where " $\mathrm{m}$ " is a mass in kilograms, "g" is the standard gravitational constant, and " $h$ " is the height in meters, which in this case is the chest displacement " $D_{c}(s)$ ". The force $(F)$ acted on the subject's chest is dependent on the mass portion of the total subject's body mass in kilograms residing at the chest area, i.e., " $\left(\mathrm{M}_{\mathrm{L}}+\right.$ $\mathrm{M}_{\mathrm{R}}$ )" and gravity " $\mathrm{g}$ ". Interestingly, Quetelet stated in his book on page 66 that "... the weight of developed persons, of different heights, is nearly as the square of the stature." and "... a transverse section, giving both the breadth and thickness, is just proportioned to the height of the individual" [8]. Therefore, it is reasonable to assume that the mass portion of the total subject's body mass in kilograms residing at the chest area, i.e., " $\left(\mathrm{M}_{\mathrm{L}}+\mathrm{M}_{\mathrm{R}}\right)$ " can be estimated using 
Quetelet's Index. At the time of Quetelet, the relationship between the body weight and height was known as Quetelet's Index, however, in 1972, it was renamed as Body Mass Index (BMI). Therefore, the constant " $K$ " in (2) can be redefined in relation to the subject's actual body mass " $\mathrm{M}$ " in kilograms, actual height " $\mathrm{H}$ " in meters, and " $\mathrm{g}$ " as the standard gravitational constant, i.e., $9.80665 \mathrm{~ms}^{-2}$ :

$$
K=g\left(M_{p L}+M_{p R}\right)=g\left(M / H^{2}\right)=g B M I \quad\left(k g m^{-1} S^{-2}\right)
$$

To determine the units for the transfer function in (2), all units need to be combined. The gravity and the chest acceleration unit is $\mathrm{ms}^{-2}$, the BMI unit is $\mathrm{kgm}^{-2}$, and the denominator "s" unit is $\mathrm{s}^{-1}$. The combination of units is $\mathrm{kgms}^{-2} \mathrm{~m}^{-2} \mathrm{~s}^{-1}$, which equal to $\mathrm{Nm}^{-2} \mathrm{~s}^{-1}$. Pressure is measured in SI unit of Pascal $(\mathrm{Pa})$, equals to $1 \mathrm{Nm}^{-2}$. Therefore, the unit for the transfer function in (2) is Pascal per second $\left(\mathrm{Pas}^{-1}\right)$.

The relationship between the intrapulmonary pressure and the chest displacement can now be defined and expressed as:

$$
\frac{d}{d t} P_{I}(t)=-g B M I\left(\frac{d^{2}}{d t^{2}} d_{c}(t)-f_{R R} d_{c}(t)\right)\left(P^{2} s^{-1}\right)
$$

For the discrete-time implementation of the pulmonary ventilation model, matched Z-transform method has been chosen for the conversion of s-domain relationships to $\mathrm{Z}$ domain, where " $\mathrm{T}_{\mathrm{s}}$ " is the sampling time in second and " $\mathrm{f}_{\mathrm{s}}$ " is the sampling rate in Hertz:

$\frac{P_{I}(z)}{D_{C}(z)}=-g B M I\left(\frac{1-k_{z} z^{-1}+z^{-2}}{1-z^{-1}}\right)$ where $k_{z}=2 \cosh \left(T_{s} \sqrt{f_{R R}}\right)$

The discrete-time instantaneous rate of change of the intrapulmonary pressure can be expressed as:

$$
\begin{aligned}
& \Delta P_{I}[n]=-g B M I f_{s}^{2}\left[d_{c}[n]-k_{z}[n] d_{c}[n-1]+d_{c}[n-2]\right]\left(\operatorname{Pas}^{-1}\right) \\
& k_{z}[n]=2 \cosh \left[T_{s} \sqrt{\frac{\text { respiratory rate }[n]}{60}}\right]
\end{aligned}
$$

The square of " $f_{s}$ " in (7) is the amplitude gain constant when differentiate in discrete-time.

Trapezoidal numerical integration over 3 sample points (due to chest acceleration) yields the discrete-time instantaneous intrapulmonary pressure:

$$
P_{I}[n]=P_{I}[n-1]-g B M I f_{S}\left[\begin{array}{c}
\left(d_{c}[n]\right) \\
\left(-\left[k_{z}[n]-1\right] d_{c}[n-1]\right) \\
\left(\left[1-k_{z}[n-1]\right] d_{c}[n-2]\right) \\
\left(d_{c}[n-3]\right)
\end{array}\right]
$$

Applying Boyle's law relationship between pressure and volume in a closed system, the discrete-time instantaneous tidal volume $\left(\mathrm{V}_{\mathrm{t}}\right)$ in milliliters, i.e., tidal value at a particular instance not the tidal volume per breath can be expressed as:

$V_{t}[n]=\left\{\begin{array}{cc}V_{T L C}\left[\frac{P_{I}[n-1]}{P_{I}[n]}-1\right], & P_{I}[n] \neq 0 \\ 0 & , \\ P_{I}[n]=0\end{array}\right\} \quad(m L)$

"V $\mathrm{TLC}_{\text {" }}$ is the subject's total lung capacity (TLC) in milliliters. Adopting the power law prediction parameters for respiratory variables in mammals by Stahl [9] and the height- weight covariance by Livingston and Lee [10], "V $\mathrm{VLC}$ " can be expressed in form of "allometric" formula, where " $\mathrm{Wt}$ " is the subject's predicted ideal body mass in kilograms and " $\mathrm{Ht}$ " is the subject's actual height in centimeters:

$$
V_{T L C}=53.5 W t^{1.06}(\mathrm{~mL}) \text { where } \mathrm{Wt}=(\mathrm{Ht} / 33.34)^{0.3922^{-1}}(\mathrm{~kg})
$$

The functional residual capacity (FRC) is unknown, and it is not required to be known, therefore, the discrete-time instantaneous tidal volume $\left(\mathrm{V}_{\mathrm{t}}\right)$ will have a reference line at 0 . This is the reason for subtracting the value of 1 in (10).

To compensate for any noises and/or unwanted body movements, the subject's predicted tidal volume $\left(\mathrm{V}_{\mathrm{pt}}\right)$ in milliliters must be calculated to be used as the artefacts rejection criteria. According to Stahl [9], Livingston and Lee [10], " $\mathrm{V}_{\mathrm{pt}}$ " can be expressed in form of "allometric" formula, where "Wt" and "Ht" as described above in relation to (11):

$$
V_{p t}=7.69 W t^{1.04}(\mathrm{~mL}) \text { where } \mathrm{Wt}=(\mathrm{Ht} / 33.34)^{0.3922^{-1}}
$$

Therefore, the discrete-time instantaneous tidal volume with the inclusion of artefact rejection criteria $\left(\mathrm{V}_{\mathrm{t}}^{*}\right)$ in milliliters can be expressed as:

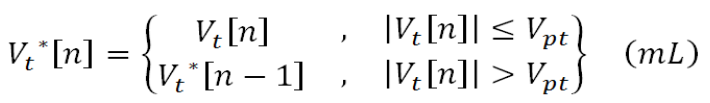

\section{Estimation Algorithm}

A block diagram of the novel intrapulmonary pressure and tidal volume estimation algorithm is presented below:

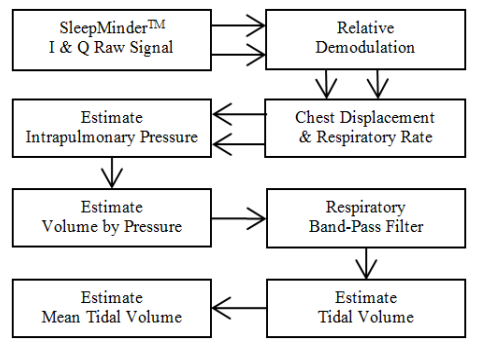

Figure 2. Intrapulmonary Pressure \& Tidal Volume Estimation Algorithm

The "Relative Demodulation" technique from [11] is used to obtain the real-time chest displacement and respiratory rate. The "Estimate Intrapulmonary Pressure" (EIP) implements the "P," equation as stated in (9). The "Estimate Volume by Pressure" (EVBP) implements the " $\mathrm{V}_{\mathrm{t}}$ ", equation as stated in (13). Butterworth band-pass filter has been chosen to implement the "Respiratory Band-Pass Filter" (RBPF). The reason for choosing such type of filter is the simplicity in implementation and faster performance as compared to other FIR filters, which is more applicable for real-time embedded applications. The selected frequency bandwidth for RBPF is $0.2-0.5 \mathrm{~Hz}$ which corresponds to $12-$ 30 breaths per minute. The "Estimate Tidal Volume" (ETV) identifies the peak locations of the estimated instantaneous " $\mathrm{V}_{\mathrm{t}}^{*}$ " waveform. One tidal cycle is determined by the current and previous identified peak of " $\mathrm{V}_{\mathrm{t}}^{*}$ ". The maximum and minimum values are then identified per cycle. The tidal volume is then estimated as the difference between the 
maximum and the minimum values of " $\mathrm{V}_{t}$ " " over a cycle. The value of " $\mathrm{V}_{\mathrm{pt}}$ " is also applied as a conditional rule, i.e., if the current estimated tidal volume is greater than " $\mathrm{V}_{\mathrm{pt}}$ ", then the current estimated tidal is equal to the previous estimated tidal volume. The "Estimate Mean Tidal Volume" (EMTV) calculates the mean value of the tidal volume per selected window-width. For this research purpose, a fixed windowwidth of 60 seconds ( 2 epochs) and a sliding window-width of 30 seconds (1 epoch) are employed.

\section{RESULT \& DISCUSSION}

In order to compare the output of the algorithm with the gold-standard PSG recordings, the PSG Pressure signal from the cannula pressure sensor sampled at $8 \mathrm{~Hz}$ was selected as the reference signal. However, since the sample rate of the PSG Pressure differs from the SleepMinder ${ }^{\mathrm{TM}}$ (SM) sample rate, the Pressure signal was first up-sampled to the SM sample rate. The tidal volume estimation for the up-sampled pressure signal was performed via EVBP, RBPF, ETV, and EMTV as described above in relation to "Fig. 2". This ensures that identical mechanisms are applied to both SM and PSG estimations for accurate comparison. The accuracy percentages, as well as errors per patient, were calculated by comparing per sample, the estimated mean tidal volume value from the SM to the PSG for the entire sleep recording duration. The resultant median accuracy percentage and error values were then obtained over the patient's entire recording duration. The final median accuracy percentage and error over the entire sample population, i.e., twenty CHF patients, were obtained via statistical analysis as shown in "Fig. 3".
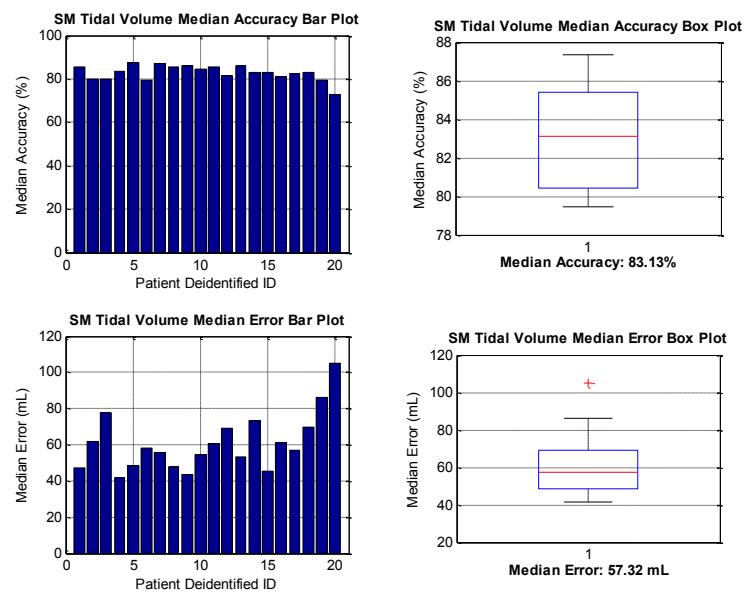

Figure 3. SleepMinder ${ }^{\mathrm{TM}}$ estimated tidal volume median accuracy \& error

The performance measures were obtained for all twenty CHF patients and are shown in "Fig. 3". Across all twenty CHF patients' recordings with mean recorded sleep duration of 7.76 hours, the tidal volume estimation median accuracy achieved $83.13 \%$ with a median error of 57.32 milliliters. The challenges that affect the accuracy of the tidal volume estimation is the accuracy of the real-time chest displacement magnitude and the respiratory rate, which are impacted by the complexity of sleep environment with unpredictable body movements, body orientation, and interferences. Undesired harmonics, body movements, body orientation, especially in the complexity of sleep environment are the current challenges in non-contact signal processing.

\section{CONCLUSION}

This paper has demonstrated the non-contact estimation of intrapulmonary pressure and tidal volume for CHF patients in the complexity of sleep environment with good accuracy. A potential application would be non-contact continuous monitoring of intrapulmonary pressure and tidal volume during sleep in the home.

It is also important to emphasize that the proposed pulmonary ventilation model is not restricted to non-contact applications; it is a novel mathematical model that can also be applied to PSG systems or home sleep test devices.

Future work will extend the implementation and verification of minute ventilation, flow rate, pulmonary compliance, and focuses on overall accuracy enhancements.

\section{ACKNOWLEDGMENT}

The authors would like to acknowledge the support of ResMed in providing the patented non-contact biomotion sensor and patients' database for this research.

\section{REFERENCES}

[1] T. Young, M. Palta, J. Dempsey, J. Skatrud, S. Webber, and S. Badr, "The Occurrence of Sleep-Disordered Breathing Among MiddleAged Adults," The New England Journal of Medicine, vol. 328, no. 17, pp. 1230-1235, 1993.

[2] P. E. Peppard, T. Young, J. H. Barnet, M. Palta, E. W. Hagen, and K. M. Hla, "Increased Prevalence of Sleep-Disordered Breathing in Adults," American Journal of Epidemiology, vol. 177, no. 9, pp. 1006-1014, 14 April, 2013, 2013.

[3] G. Amabile, F. Pauri, and F. Fattapposta, "Neurological Correlations in Neurological Breathing Disorders," Surgery for Snoring and Obstructive Sleep Apnea Syndrome, M. Fabiani, ed., pp. 39-46, The Netherlands: Kugler Publications, 2003.

[4] H. K. Yaggi, J. Concato, W. N. Kernan, J. H. Lichtman, L. M. Brass, and V. Mohsenin, "Obstructive Sleep Apnea as a Risk Factor for Stroke and Death," The New England Journal of Medicine, vol. 353, no. 19, pp. 2034-2041, 10 November, 2005, 2005.

[5] R. S. T. Leung, and T. D. Bradley, "Sleep Apnea and Cardiovascular Disease," American Journal of Respiratory and Critical Care Medicine, vol. 164, no. 12, pp. 2147-2165, 2001.

[6] Y. S. Lee, P. N. Pathirana, C. L. Steinfort, and T. Caelli, "NonContact Measurement of Respiratory Function and Deduction of Tidal Volume," in 36th Annual International Conference of the IEEE Engineering in Medicine and Biology Society, Chicago, Illinois, USA, 2014, pp. 594-597.

[7] W. Massagram, N. Hafner, V. Lubecke, and O. Boric-Lubecke, "Tidal Volume Measurement Through Non-Contact Doppler Radar With DC Reconstruction," IEEE Sensors Journal, vol. 13, no. 9, pp. 3397-3404, 2013.

[8] A. Quetelet, A treatise on man and the development of his faculties, Edinburgh, Scotland: William and Roberts Chambers, 1842.

[9] W. R. Stahl, "Scaling of respiratory variables in mammals," Journal of Applied Physiology, vol. 22, no. 3, pp. 453-460, 1967.

[10] E. H. Livingston, and S. Lee, "Body surface area prediction in normal-weight and obese patients," American Journal of Physiology - Endocrinology and Metabolism, vol. 281, no. 3, pp. E586-E591, 2001.

[11] V. P. Tran, and A. A. Al-Jumaily, "Non-Contact Dual Pulse Doppler System Based Real-Time Relative Demodulation and Respiratory \& Heart Rates Estimations for Chronic Heart Failure Patients," Procedia Computer Science, vol. 76, no. 2015, pp. 47 - 52, 2015. 